\title{
Schmallenberg virus reveals its secrets
}

Schmallenberg virus (SBV) emerged in central and western Europe in 2011 and causes abortion, stillbirth and congenital malformations in newborn ruminants. Now, two groups report the development of an efficient genetic system to recover the virus from cloned cDNA, as well as the results of initial investigations into the pathogenesis of this new virus and how it interacts with the host innate immune system.

$\mathrm{SBV}$ is a member of the

Orthobunyavirus genus in the Bunyaviridae family and has a tripartite, single-stranded negative-sense RNA genome. The genome sequence of SBV was determined in 2011 and is publicly available in GenBank; however, certain features, such as the terminal consensus nucleotides, were missing from the deposited sequence, and there were some suspected artefacts. SBV belongs to the Simbu serogroup of orthobunyaviruses,

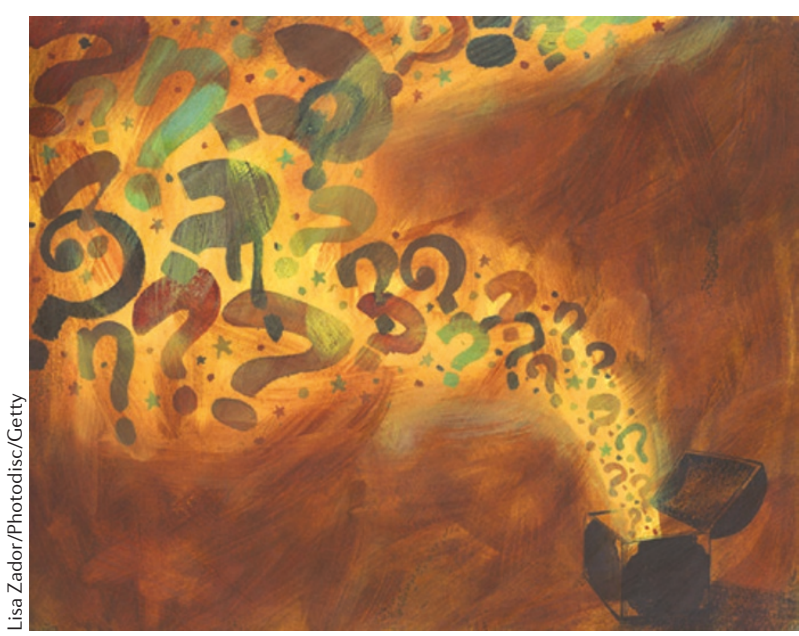

and both teams therefore made use of the available sequences for other Simbu viruses to infer the correct full-length sequence for SBV. Other members of the Bunyaviridae family have been rescued from cloned cDNA (an approach that is known as reverse genetics) using a T7 RNA polymerase-driven plasmid-based technique that was first developed for Bunyamwera virus, the prototypical orthobunyavirus. Elliott and colleagues used reverse transcription PCR (RT-PCR) to amplify the three SBV genome segments and then cloned the resulting PCR products into T7 transcription plasmids; Varela and colleagues took a synthetic approach, and in this study the three SBV cDNA-containing plasmids were synthesized commercially. Following transfection of a cell line that constitutively expresses T7 RNA polymerase, both groups were able to isolate the virus, and detailed characterization revealed that there were no differences between the rescued viruses and natural isolates. Varela et al. also developed an alternative rescue system in which T7 RNA polymerase was transiently co-expressed.

In other orthobunyaviruses, including Bunyamwera virus and La Crosse virus, it is known that one of the non-structural proteins, NSs, inhibits host transcription, thus blocking the synthesis of type I interferon (IFN). To establish whether this protein has a similar role in SBV infection, both groups generated SBV mutants lacking the
NSs protein by introducing stop codons in the NSs ORF at positions that would not alter the sequence of the overlapping nucleocapsid $(\mathrm{N})$ protein. Cells infected with wild-type virus produced little IFN, whereas IFN production was significantly increased in cells infected with SBV that lacked NSs, indicating that NSs is an IFN antagonist. In an RT-PCR assay, Varela et al. could detect IFNB mRNA in cells infected with the NSs-negative SBV but not in cells infected with wild-type SBV. Finally, Varela et al. went on to establish a mouse model of infection (using NIH-Swiss mice), which allowed a detailed analysis of the progression of necrosis and haemorrhage in the brain over the course of the infection. Histopathological and immunohistochemical analyses of tissue sections from lambs and calves that had been naturally infected in utero revealed that SBV replicates in neurons in the brain and spinal cord.

The SBV outbreak has had a devastating economic impact, and there is great interest in the development of a vaccine to tackle this emerging disease. The development of an efficient reverse genetics system for SBV is a crucial first step towards this goal.

ORIGINAL RESEARCH PAPERS Elliott, R. M. et al. Establishment of a reverse genetic system for Schmallenberg virus, a newly emerged orthobunyavirus in Europe.J. Gen. Virol. 19 Dec 2012 (doi:10.1099/vir.0.049981-0) | Varela, M. et al. Schmallenberg virus pathogenesis, tropism and interaction with the innate immune system of the host. PLoS Pathog. 9, e1003133 (2013) 